\title{
Harmonic current injection for torque ripple reduction with optimum current trajectory for minimum induced voltage
}

\author{
Andreas Langheck \\ Karlsruher Institut of Technology (KIT) \\ Institute of Electrical Engineering \\ Karlsruhe, Germany \\ andreas.langheck@kit.edu \\ https://orcid.org/0000-0001-9309-2781 \\ Thorsten Rittgerott \\ Schaeffler Automotive Buehl GmbH \& \\ Co. $K G$ \\ Buehl, Germany
}

\author{
Dominik Stretz \\ Schaeffler Technologies $A G \&$ Co. $K G$ \\ Herzogenaurach, Germany \\ stretdmi@schaeffler.com
}

Johannes Kolb

Schaeffler Technologies $A G$ \& Co. $K G$

Karlsruhe, Germany

johannes.kolb@schaeffler.com

\author{
Jie Zhou \\ Schaeffler Automotive Buehl GmbH \& \\ Co. $K G$ \\ Buehl, Germany \\ Jie.Zhou@schaeffler.com
}

\author{
Martin Doppelbauer \\ Karlsruher Institut of Technology (KIT) \\ Institute of Electrical Engineering) \\ Karlsruhe, Germany \\ martin.doppelbauer@kit.edu
}

\begin{abstract}
Electric drives often do not have a smooth torque. In most cases they are coupled with mechanical systems. These tend to have natural frequencies. Therefore, it is advisable to eliminate harmonics from the torque pulsation if they are close to a natural frequency. One possibility to do this is harmonic current injection or HCI for short. However, the question arises, how exactly this harmonic current should be. The fact that a harmonic in the torque can be influenced by the $d$ and $q$ current results in a greater degree of freedom. This paper presents a method to investigate all possible solutions. Furthermore, two optimization possibilities for the current trajectory are presented. The effect of this selection on the maximum torque speed curve is shown. It has been found that the method which minimizes the induced voltage achieves an up to $8 \%$ larger range of application in this example.
\end{abstract}

Keywords-PMSM, Harmonic current injection, voltage optimal current trajectory, torque ripple

\section{INTRODUCTION}

Synchronous AC Machines with permanent magnet (PMSM) excitation are widespread. They convince with high power/ torque density and high efficiency. Therefore, various applications such as household appliances, industrial applications, aviation applications, electric and hybrid vehicles use PMSMs. As a matter of principle, these machines often come along with a non-smooth torque. The torque which deviates from the average value is called pulsating torque [1].

As large as the variety of different PMSMs is the number of causes for torque ripples. Especially the electromagnetic design has an influence on the torque ripple. Different rules apply for smoother torque for example the selection of stator slots and pole pairs [2].Choosing the right winding with high fundamental and low harmonics is essential [3]. As well as detailed optimization of the geometry such as on the tooth tips of the stator or the magnet pole overlapping [2].

Although the mechanisms of action are known and taken into account in the design of the electric machines, they have a non-smooth torque. On the one hand, this is due to the fact that there are limits to the implementation of the measures, on the other hand, it is also due to the fact that the tradeoff between costs and power density must always be found in the economy. High power density results in higher saturation induced pulsation [4]. Economical winding techniques take their toll with poorer fundamental to harmonic ratios or poorer magnetic tooth shapes [5].

In this paper we investigate the possibility to reduce torque pulsations with additional harmonic current injections (HCI) to the regular controlling current. Several investigations have been done before. In general, this technique modifies the current in a way that reduces torque pulsation. Some publications reduce all torque ripples within a small band around the average torque and use numerical or binary search method together with Finite Element Analysis (FEA) to set the corresponding current [6]. Other publications focus on the $(k-1)^{\text {th }}$ and/or $(k+1)^{\text {th }}$ electrical harmonics in the current in order to distinguish the $k^{\text {th }}$ electrical harmonic in the torque [7][8].

The above mentioned publications do not deal with the exact choice of harmonics. As it turned out during investigations, the assignment between harmonics in the current and suppression in the torque is not unique, but leaves a certain degree of freedom. The specific damping of a harmonic in the torque pulsation can be achieved by different current trajectories. This degree of freedom can be used to fulfill certain constraints. This paper proposes a method for finding a trajectory best suited to a given optimization objective.

\section{PUlsating TORQue}

According to [1] the pulsating torque is defined as the sum of cogging torque and ripple torque. The former is generated by the interaction of the magnetic flux of the rotor and angular changes in the magnetic reluctance of the stator. By definition, no stator excitation is involved in the generation of the cogging torque.

The ripple torque is generated by the interaction between the magneto motive forces of the stator current and the electromagnetic properties of the rotor and can take two forms: a) mutual torque and b) reluctance.[1] The pulsations harmonics in three phase machines are multiple of 6 [7].

\section{A. Model description with troque ripple}

To calculate the effect of HCI on the torque, a model is needed which calculates the torque dependent on the rotor angle $\gamma_{\mathrm{r}}$. In [9] the torque is defined as: 
$M=\frac{3}{2} p\left[\left(\psi_{\mathrm{d}}+\frac{\mathrm{d}}{\mathrm{d} \gamma_{\mathrm{r}}} \psi_{\mathrm{q}}\right) i_{\mathrm{q}}-\left(\psi_{\mathrm{q}}-\frac{\mathrm{d}}{\mathrm{d} \gamma_{\mathrm{r}}} \psi_{\mathrm{d}}\right) i_{\mathrm{d}}\right]+M_{\text {stray }}$

In Equation (1) $M$ is the torque, $i_{\mathrm{d}}$ and $i_{\mathrm{q}}$ are the direct and quadrature current in the rotating rotor frame. $\psi_{\mathrm{d}}$ and $\psi_{\mathrm{q}}$ holds the current and angle dependent flux linkage. Effects like cogging torque and every torque pulsating which is not sensed by the winding of the machine is summed up in $M_{\text {stray }}$ and therefore also current and angle dependent. An efficient and comfortable way of handling angular dependencies and to avoid problems with the discretization of $\gamma_{\mathrm{r}}$ is to use Fourier series [10]. For example:

$\psi_{x}\left(\gamma_{\mathrm{r}}\right)=\frac{a_{x, 0}}{2}+\sum_{n=1}^{N}\left(\psi_{x, a, n} \cos (n \omega t)+\psi_{x, b, n} \sin (n \omega t)\right)$

Where $x$ is the placeholder for either direct or quadrature (d / q) component, $\omega$ is the synchronous electrical frequency with $\omega t=\gamma_{\mathrm{r}}$. Fourier series can also be applied on $M_{\text {stray }}$. Furthermore, from above is known that with ideal smooth current $i_{\mathrm{d}}$ and $i_{\mathrm{q}}$ only the fundamental and harmonics of the $6 k^{\text {th }}$ order are present. This means for (2) $n=6 k$ with $k=$ $1,2,3, \ldots$

In this paper all physical quantities for the parameterization of the model were calculated by FEA. The difference between the torque produced by the multiplication of the flux linkage and the current and the torque calculated with the virtual work principle in the FEA is included in $M_{\text {stray }}$. The torque $M_{\text {stray }}$ calculated in this way is averagefree and a possibility to model the cogging torque. In Fig. 1. the three torque components are shown. This Figure shows that when applying HCI, attention must also be paid to the torque component $M_{\text {stray }}$, which cannot be expressed by the flux linkage.

It is also possible to parametrize this model with flux linkage obtained from a measurement [10], [11]. To measure and model $M_{\text {stray }}$ special methods are needed [12].

\section{B. Torque ripple example}

The PMSM under investigation is designed as a hybrid machine for the traction sector. It is produced with a distributed windings in star connection and has a maximum power of $150 \mathrm{~kW}$. The distribution of the fundamental torque and the unwanted $6^{\text {th }}$ harmonic of the pulsating torque is depicted in Fig. 2. In addition, the operation point at
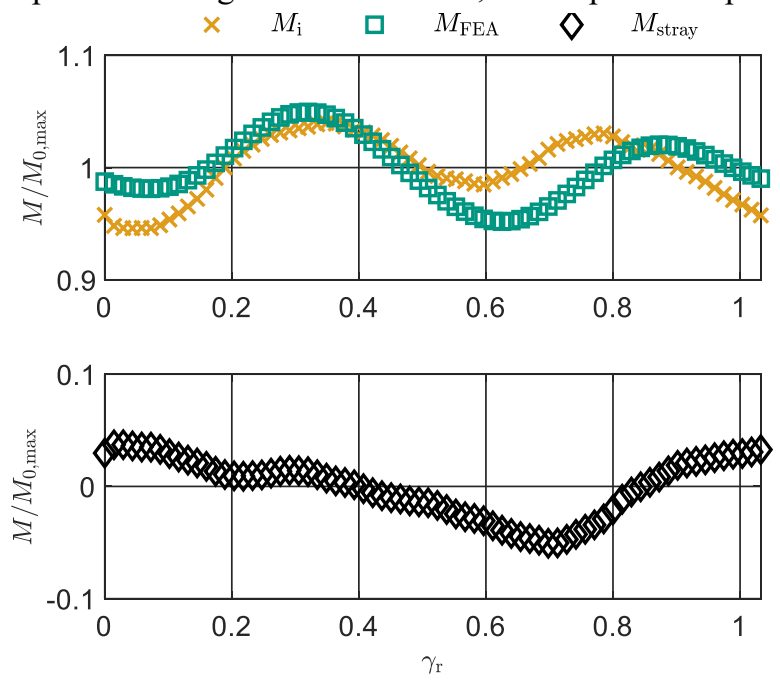

Fig. 1: Angle dependent course of the inner torque $M_{i}$, torque from FEA $M_{F E A}$ and $M_{\text {stray }}$ at marked operation point characteristic speed is plotted in red. Up to this point the Maximum Torque Per Ampere (MTPA) strategy is applied and the harmonic part is limited. The higher the speed, the more you have to weaken the field and the more the harmonic part grows. This relationship is double disadvantageous, because the fundamental torque decreases and therefore the $M_{0} / M_{6}$ ratio gets worse quickly. The same applies to the higher harmonics.

With HCI, the harmonic portion of the torque is to be reduced. At the same time, it can be seen in Fig. 2 that HCI will be mainly used when field weakening occurs. Therefore, a method is necessary which still works near the voltage limit.

\section{HARMONIC CURRENT}

In the following a harmonic current is used to eliminate a harmonic torque. However, the presented procedure is not limited to this or to this choice of harmonics.

Because we want to eliminate pulsating torque, which occurs at the $6 k^{\text {th }}$ order we focus on that. The needed current can be described in stator frame orientation as $( \pm 6 k+1)^{\text {th }}$ or in rotor oriented frame as $6 k^{\text {th }}$ harmonic order in direct or quadrature direction. When the phase angle of the injected torque relative to the pulsating torque is $\pm \pi$, the pulsating torque component will vanish.

\section{A. Effects of HCI on torque}

PMSMs are current controlled, in real application as well as in FEAs or in this model. Therefore, we start with the definition of a general harmonic current in rotor oriented frame:

$$
\begin{aligned}
& i_{\mathrm{d}}(t)=i_{\mathrm{d} 0}+\sum_{n}^{N_{n}} i_{\mathrm{d}, n} \cos \left(n \omega t+\varphi_{\mathrm{d}, n}\right) \\
& i_{\mathrm{q}}(t)=i_{\mathrm{q} 0}+\sum_{n}^{N_{n}} i_{\mathrm{q}, n} \cos \left(n \omega t+\varphi_{\mathrm{q}, n}\right)
\end{aligned}
$$

In (3) and (4) the operation point of the PMSM is controlled with $i_{\mathrm{d} 0}$ and $i_{\mathrm{q} 0}$. The current oscillating around the operation point with the frequency $n \omega$, the amplitudes $i_{\mathrm{d}, n} /$ $i_{\mathrm{q}, n}$ and the phases $\varphi_{\mathrm{d}, n} / \varphi_{\mathrm{q}, n}$ produces a harmonic torque. $N_{n}$ is the quantity of injected harmonics and $\omega$ is the synchronous electrical frequency of the PMSM. As described above:

$$
n=6 k \quad \text { with } \quad k=1,2,3, \ldots
$$

applies.

If we simplify (1) and neglect the derivation by angle and express the flux linkages by their inductivities $\psi_{\mathrm{d}}=i_{\mathrm{d}} L_{\mathrm{d}}+$ $\psi_{\mathrm{PM}}$ and $\psi_{\mathrm{q}}=i_{\mathrm{q}} L_{\mathrm{q}}$, we get:
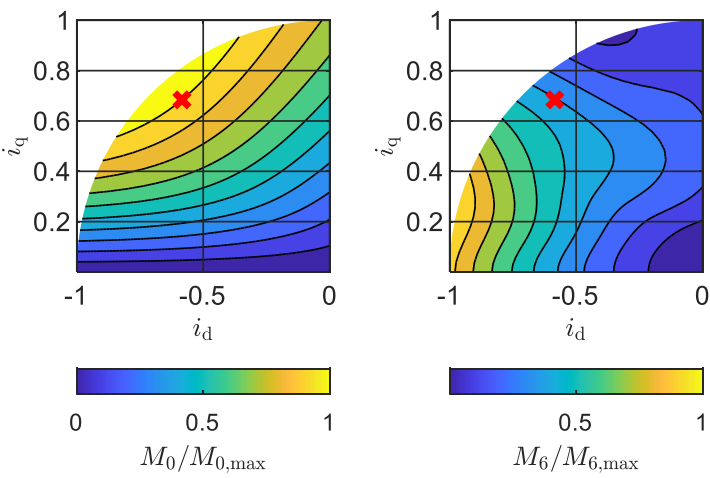

Fig. 2: Distribution of fundamental torque (left), pulsating torque of order 6 (right), over current plane with operation point (red) 


$$
M=\frac{3}{2} p\left[\psi_{\mathrm{PM}} i_{\mathrm{q}}+\left(L_{\mathrm{q}}-L_{\mathrm{d}}\right) i_{\mathrm{q}} i_{\mathrm{d}}\right]
$$

This procedure is acceptable in order to investigate the basic mechanisms of action. In the actual HCI algorithm, described below, we consider both angle and current dependencies.

With only one injected harmonic current in direct and quadrature direction (3) and (4) in (7) forms three parts, which can be described as following:

$$
\begin{gathered}
M_{0}=\frac{3}{2} p\left[\psi_{\mathrm{PM}} i_{q 0}+\right. \\
\left(L_{\mathrm{d}}-L_{\mathrm{q}}\right)\left(i_{\mathrm{d} 0} i_{\mathrm{q} 0}+i_{\mathrm{d} n} i_{\mathrm{q} n} \frac{1}{2} \cos \left(\varphi_{\mathrm{d} n}\right.\right. \\
\left.\left.\left.-\varphi_{\mathrm{q} n}\right)\right)\right]
\end{gathered}
$$

From (8) - (10) we can see:

- In general (if $\varphi_{\mathrm{d} n} \neq \varphi_{\mathrm{q} n}$ and $L_{\mathrm{d}} \neq L_{\mathrm{q}}$ ) a harmonic current results in an addition fundamental torque for $i_{\mathrm{d} n} \neq 0$ and $i_{\mathrm{q} n} \neq 0$

- A harmonic torque with the order of $n$ can be produced with a harmonic current in quadrature direction and if $L_{\mathrm{d}} \neq L_{\mathrm{q}}$ and $i_{\mathrm{q} 0} \neq 0$ also with a harmonic current in direct direction.

- The use of direct and quadrature components results in a torque with the double frequency $2 n \omega$

- If there are more than one injected harmonics additional terms in $M_{0}$ and combinations of different harmonics will occur.

In summary, a harmonic current of order $n$ contributes a certain part to the fundamental torque and generates a harmonic torque of equal and double order. $M_{n}$ is described by (9) and contains four unknown variables: $i_{\mathrm{d}, n}, i_{\mathrm{q}, n}, \varphi_{\mathrm{d}, n}$ and $\varphi_{\mathrm{q}, n}$. With (3) and (4) these four unknown variables describe a current oscillation around an operating point. This oscillation can be understood as a trajectory, which generally describes an ellipse, if only one harmonic torque is injected. With several harmonics this ellipse becomes a Lissajous curve.

\section{B. Transformation to ellipse representation}

All possible current trajectories can be described mathematically as ellipses. In this way the direction and the bulge of the ellipse can be described separately. Therefore (3) and (4) are represented as:

$\left[\begin{array}{l}i_{\mathrm{d}} \\ i_{\mathrm{q}}\end{array}\right]=\left[\begin{array}{l}i_{\mathrm{d} 0} \\ i_{\mathrm{q} 0}\end{array}\right]+\left[\begin{array}{cc}\cos [\gamma] & -\sin [\gamma] \\ \sin [\gamma] & \cos [\gamma]\end{array}\right] \cdot\left[\begin{array}{c}\frac{i_{n}}{\sqrt{1+\alpha^{2}}} \cos \left(n \omega t+\varphi_{\mathrm{c}}\right) \\ \frac{i_{n} \alpha}{\sqrt{1+\alpha^{2}}} \sin \left(n \omega t+\varphi_{\mathrm{c}}\right)\end{array}\right]$

For the sake of simplicity, the following will only deal with one harmonic. In (11) $\gamma$ forms a rotational matrix with the trigonometrical functions which describes the direction of the ellipse. $\alpha$ describes the bulge of the ellipse, $i_{n}$ describes the amplitude of the oscillation. The starting point of the ellipse or the offset to the rotor axis is expressed with the common phase $\varphi_{\mathrm{c}}$.

Fig. 3 clarifies the function of $\gamma$ and the term "direction". Exemplary shown are three values for $\gamma=\left[0, \frac{\pi}{2}, \frac{3 \pi}{4}\right]$. The current trajectory oscillates with no bulge around a fictitious operation point of $i_{\mathrm{d} 0}=-100 \mathrm{~A}$ and $i_{\mathrm{q} 0}=100 \mathrm{~A}$ with an amplitude of $i_{n}=10 \mathrm{~A}$. The angle $\gamma$ rotates the direction starting from the d-axis mathematically positive and is defined for $\gamma \in[0, \pi]$.

Fig. 4 shows the function $\alpha$ and explains the term "bulge". Exemplary shown are three values for $\alpha=[0,0.5,1]$. The current trajectory oscillates in direction $\gamma=\frac{3 \pi}{4}$ around the same operation point. With $\alpha=0$ the ellipse is only a line and becomes a perfect circle with $\alpha=1$, therefore $\alpha \in[0,1]$ applies.

The common phase $\varphi_{\mathrm{c}}$ is explained in Fig. 5. It does not change the trajectory, but shifts its starting point. On the right side of Fig. 5 this phase offset can be well observed.

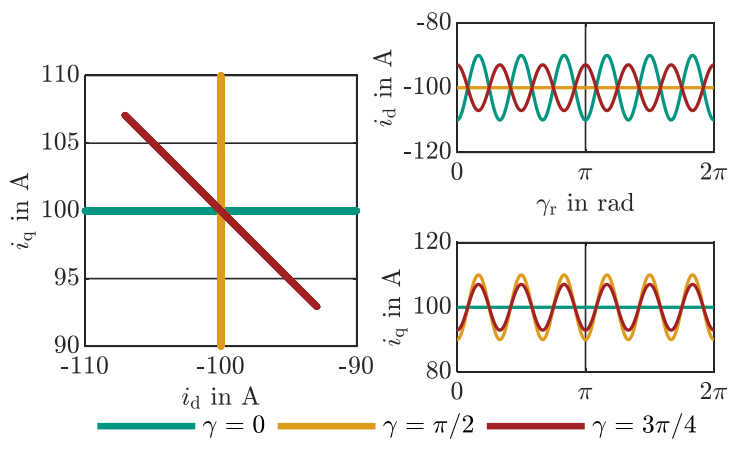

Fig. 3: Direction of ellipse display in $d q$-plane around operation point (left) and angle dependent course (right)

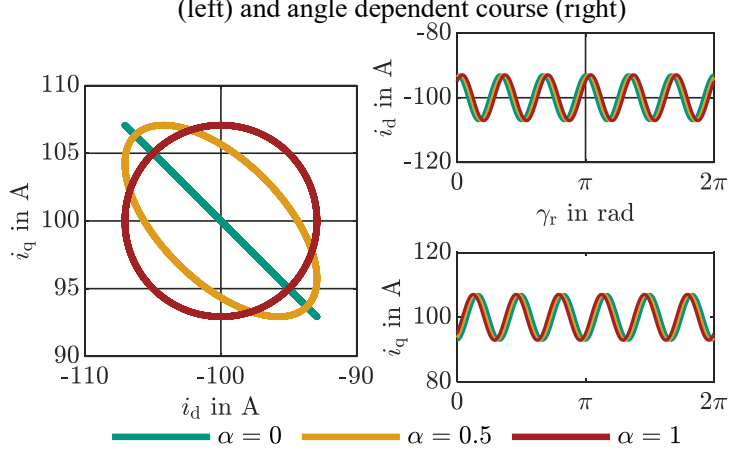

Fig. 4: Bulge of ellipse display in dq-plane around operation point (left) and angle dependent course (right)

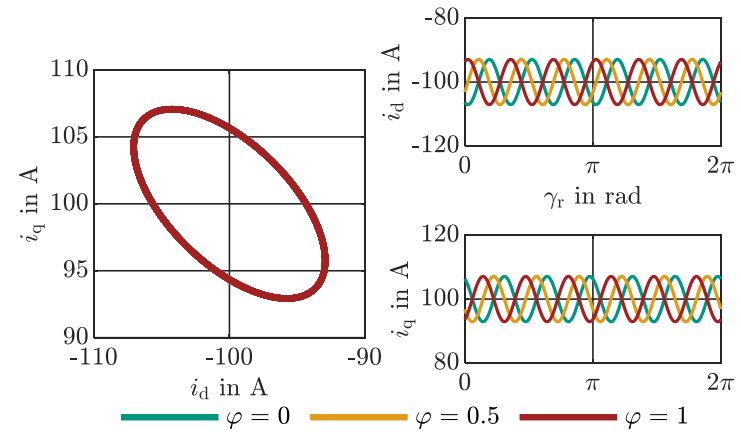

Fig. 5: Common phase of ellipse display in dq-plane around operation point (left) and angle dependent course (right) 


\section{Constant harmonic current amplitude}

The defined variable direction $\gamma$ and bulge $\alpha$ opened up a plane that can map all possible trajectories of the harmonic current. To make the influence of these two variables visible, the amplitude $i_{n}=10 \mathrm{~A}$, the operation point $i_{\mathrm{d} 0}=-100 \mathrm{~A}$, $i_{\mathrm{q} 0}=100 \mathrm{~A}$, the common phase $\varphi_{\mathrm{c}}=0$, the harmonic order $n=6$ and the speed $n_{\text {rpm }}=6000 \mathrm{rpm}$ are kept constant. The method is applied to a fictitious linear machine with $L_{\mathrm{d}}=$ $0.00057 \mathrm{H}, L_{\mathrm{q}}=0.0019 \mathrm{H}, \psi_{\mathrm{PM}}=0.0973 \mathrm{Vs}, R=0 \Omega$, $p=4$

To be able to calculate the resulting voltage the model from section III.A is extended by the corresponding equations [10]:

$$
\begin{gathered}
u_{\mathrm{d}}=R i_{\mathrm{d}}+\frac{\mathrm{d}}{\mathrm{d} t} \psi_{\mathrm{d}}-\omega \psi_{\mathrm{q}} \\
u_{\mathrm{q}}=R i_{\mathrm{q}}+\frac{\mathrm{d}}{\mathrm{d} t} \psi_{\mathrm{q}}+\omega \psi_{\mathrm{d}} \\
u_{\mathrm{s}}=\sqrt{u_{\mathrm{d}}^{2}+u_{\mathrm{q}}^{2}}
\end{gathered}
$$

In (12) and (13) the flux linkage is expressed as described in (2) or in the linear case using the inductances.

In Fig. 6 the results of all possible trajectories described as ellipse (11) are shown. The first contour plot illustrates the influence of $\mathrm{HCI}$ on $M_{0}$. For $0 \leq \gamma<\frac{\pi}{2}$ the injected current is mainly in the direct direction and fundamental torque is minimally reduced. For $\frac{\pi}{2}<\gamma \leq \pi$ the injected current is mainly in the quadrature direction and fundamental torque is minimally increased. For $\gamma=\frac{\pi}{2}$ only the quadrature direction is injected and $M_{0}$ is not changed. Overall the influence is negligible for this example.

The second contour plot in Fig. 6 illustrates the resulting $6^{\text {th }}$ harmonic torque. In accordance with the MTPA strategy a maximum harmonic torque per harmonic current can be found. In this example, this point falls within the range in which $M_{12}$ is maximum.

The maximum space vector voltage $u_{\mathrm{s}}$ for one electrical period according to (14) is shown in the 4th plot. Unlike in the other plots the maximum voltage shows no symmetry to the $y$-axis and a minimum can be found for $\alpha<0$. It is worth mentioning that this minimum does not coincide with the minimum in $M_{6}$. This leads to the conclusion that it is possible to find a trajectory with a specific harmonic torque at a minimum voltage. To do so, a $\gamma \alpha$-plane with constant harmonic torque is necessary.

\section{Constant harmonic torque}

In the case of a linear PMSM one can calculate the resulting $M_{n}$ with (9). For PMSMs with nonlinearities and angular dependencies the proposed method finds for every trajectory expressed with given $\gamma$ and $\alpha$ the corresponding amplitude $i_{n}$ and common phase $\varphi_{\mathrm{c}}$ resulting in the desired harmonic torque. Simplified, the torque from (9) can be expressed as:

$$
M_{n}^{*}=m_{n}^{*} \cos \left(n \omega t+\varphi_{n}^{*}\right)
$$

In (15) the target amplitude of the $n^{\text {th }}$ harmonic torque is $m_{n}^{*}$ which oscillates with the frequency $n \omega$ and a target phase shift of $\varphi_{n}^{*}$. In general, this can be any value, but in the following the values from Fig. 2 (right) but with reversed phase are used. By this way the harmonic torque with $n=6$ is eliminated.

Since we are confronted with a non-linear machine, $i_{n}$ and $\varphi_{\mathrm{c}}$ are found in an iterative calculation. This contains guessing an initial value for $i_{n, 0}$ and $\varphi_{\mathrm{c}, 0}$, calculate the torque according to (1) and improve the initial value for the next iteration according to:

$$
\begin{aligned}
i_{n, h+1} & =\frac{m_{n, h}}{m_{n, h}^{*}} i_{n, h} \\
\varphi_{c, h+1} & =\frac{\varphi_{n, h}}{\varphi_{n, h}^{*}} \varphi_{c, h}
\end{aligned}
$$

In (16) and (17) $m_{n, h}$ and $\varphi_{n, h}$ are the torque amplitude and phase of harmonic order $n$, in this example $n=6$, of the current iteration $h$. In case of a non-linear machine $M_{n}$ can not be calculated with (9). It is calculated as time / angle series
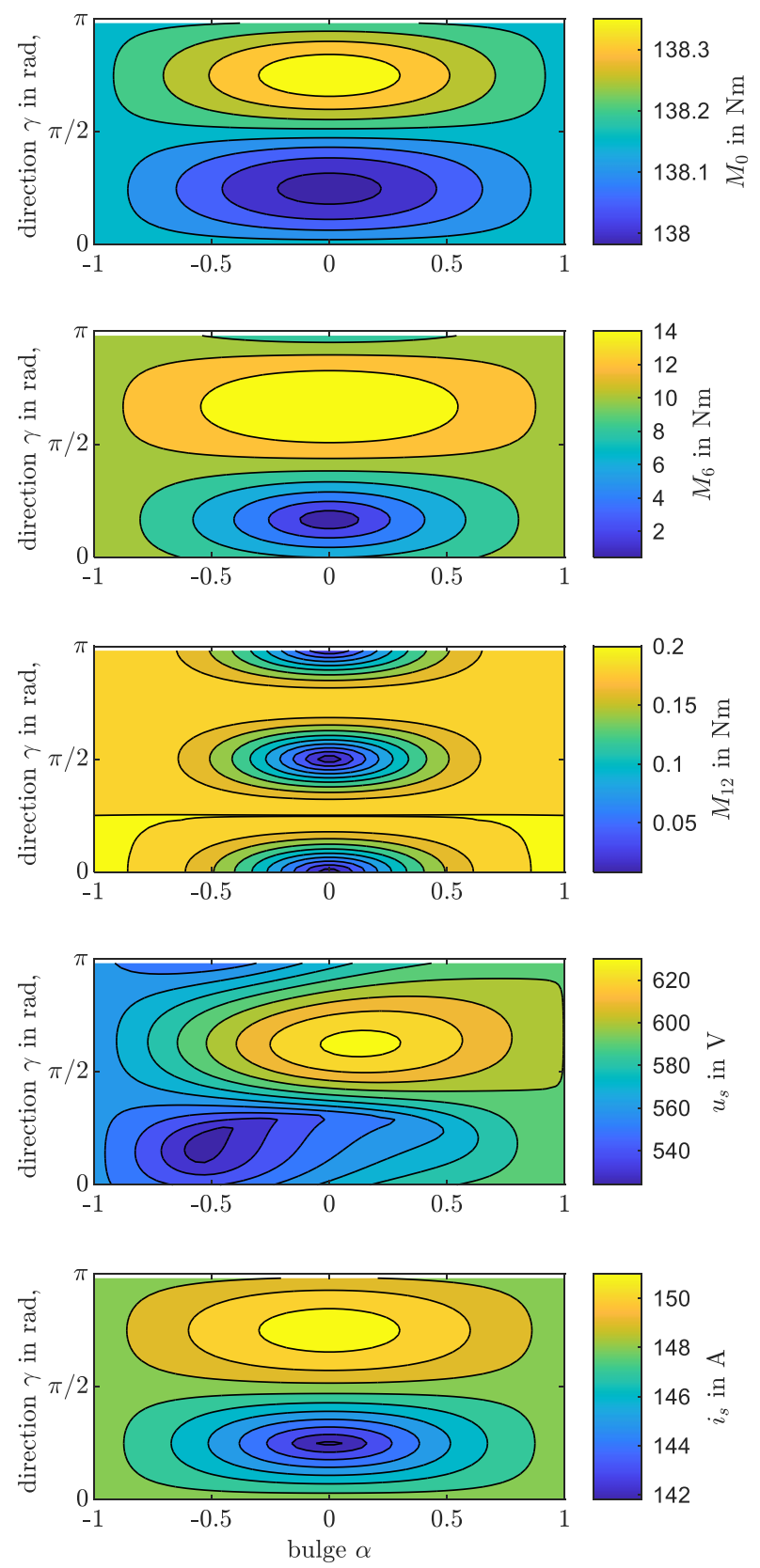

Fig. 6: Example of a $\gamma \alpha$-plane showing $M_{0}, M_{6}, M_{12}, \max \left\{u_{\mathrm{s}}\right\}, \max \left\{i_{\mathrm{s}}\right\}$ (from top to bottom) for every possible trajectory with constant current amplitude 
with (1) and (2) and afterwards analyzed with a FFT to obtain the $n^{\text {th }}$ harmonic.

Fig. 7 and Fig. 8 show an example of the iterative process (right) for the above mentioned operating point. After each iteration the figures show the resulting harmonic torque $M_{6, h}$ or $M_{6, h}+M_{12, h}$ in relation to $M_{6, h=0}$. Within 3 or 4 steps the appropriate current could be found and the resulting harmonic was eliminated good enough for the termination criterion. The proposed method works for every desired trajectory (left). In contrast to Fig. 7, Fig. 8 shows that this method also works for several harmonics simultaneously.

The proposed method uses the model described with (1) and (2), but it could also be adopted to be used directly with a FEA model of the PMSM. This might result in more exact results but also in longer simulation times. If only a trajectory at one operating point is of interest this may still be practical. However, if the entire $\gamma \alpha$-plane is to be searched for a possible optima and for all operating points, the simulation time increases rapidly.

Nevertheless, the model can only be used if it is a good approximation of the actual physics. The resulting voltages from the three current trajectories are shown in Fig. 9. (top) normalized with $u_{\mathrm{s}}$ without HCI. The solid line represents the stator voltage calculated with the model (2) and (12) - (14). The counterpart in crosses is calculated with FEA. The voltage is applied according to the rotor angle. If in the following the maximum voltage is mentioned, the maximum voltage according to the rotor angle is meant. Because of the 60 degree symmetry of the PMSM $\gamma_{\mathrm{r}} \in\left[0, \frac{\pi}{3}\right]$ applies. The color coding corresponds to that shown in Fig. 7. The corresponding torque is normalized with $M_{6}$ without $\mathrm{HCI}$ and shown in the frequency range in Fig. 9. (bottom). In addition to the

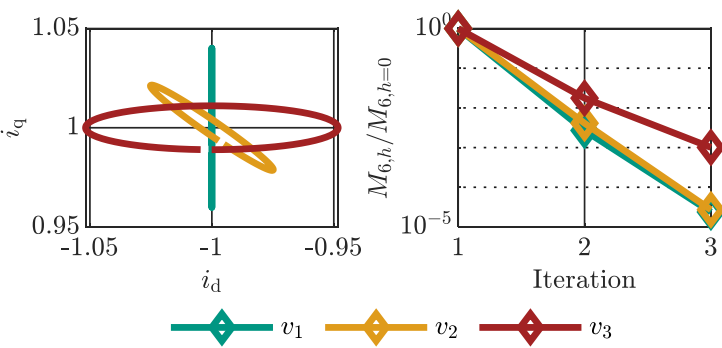

Fig. 7: Iterative HCI using the above mentioned PMSM for three different trajectories (left: $v_{1}:\left\{\gamma=\frac{\pi}{2}, \alpha=0\right\}, v_{1}:\left\{\gamma=3 \frac{\pi}{4}, \alpha=0.1\right\}, v_{1}:\{\gamma=$ $\pi, \alpha=0.25\}$ ), they have in common that the desired $6^{\text {th }}$ harmonic in torque is eliminated (right)

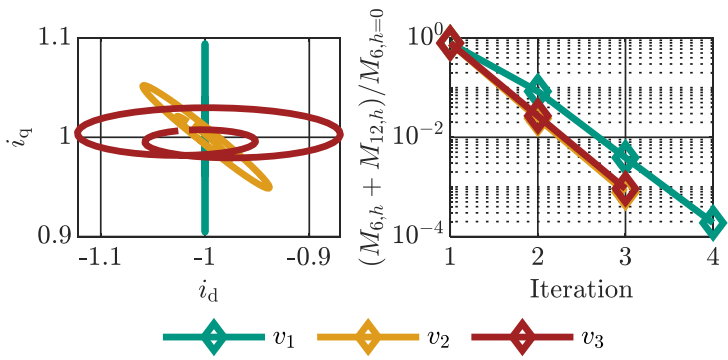

Fig. 8: Iterative HCI using the above mentioned PMSM for three different trajectories (left: $v_{1}:\left\{\gamma=\frac{\pi}{2}, \alpha=0\right\}, v_{1}:\left\{\gamma=3 \frac{\pi}{4}, \alpha=\right.$

$\left.0.1\}, v_{1}:\{\gamma=\pi, \alpha=0.25\}\right)$, they have in common that the desired $6^{\text {th }}$ $+12^{\text {th }}$ harmonic in torque is eliminated (right) proposed $\mathrm{HCI}$ trajectories the original torque $\operatorname{ref}_{\mathrm{FEM}}$ is shown. The following conclusions can be drawn from this illustration:

- The voltage and torque model fits very well to the FEA. The error is less than 4 percent in this example.

- The information on torque and voltage obtained with the model can also be reproduced in the FEA.

- This means that it is possible to search for the voltage optimum in the $\gamma \alpha$-plane with the model. Considering the many possibilities to be investigated, this results in a speed advantage.

Using this method, the $\gamma \alpha$-plane can now be set up. $\gamma$ and $\alpha$ are discretized in 52 and 51 values. For every combination of $\alpha$ and $\gamma$ the proposed HCI algorithm and voltage calculations are carried out. It should be noted that, compared to Fig. 6, all trajectories in $\gamma \alpha$-plane now eliminate the $6^{\text {th }}$ harmonic in torque. The resulting maximum voltage, normalized with $u_{\mathrm{s}}$ without $\mathrm{HCI}$, and the used harmonic current, normalized with the current amplitude of the operation point, is shown in Fig. 10.

At first glance, the white areas stand out. Here there are only solutions which are sorted out because of too high harmonic current demand. The requested trajectory is tangential to the torque contour line from Fig. 2 (left) and a lot of current is needed to have an effect on the torque. It is obvious that this area is operation point dependent.

The point of minimum voltage (marked in Fig. 10 with red cross) lies within the permitted range. A point with minimum current amplitude can also be detected (not marked). In general, these points do not coincide and must be found for every operation point.

\section{HCI FOR VARIABLE SPEED APPLICATIONS}

The described procedure can be repeated for any point in the dq-plane. Instead of simulating 52 times 51 points in the $\gamma \alpha$-plane for each operating point, an optimizer is used here. The procedure is as follows: for each operating point in the dq-plane an optimum is searched for in the $\gamma \alpha$-plane.
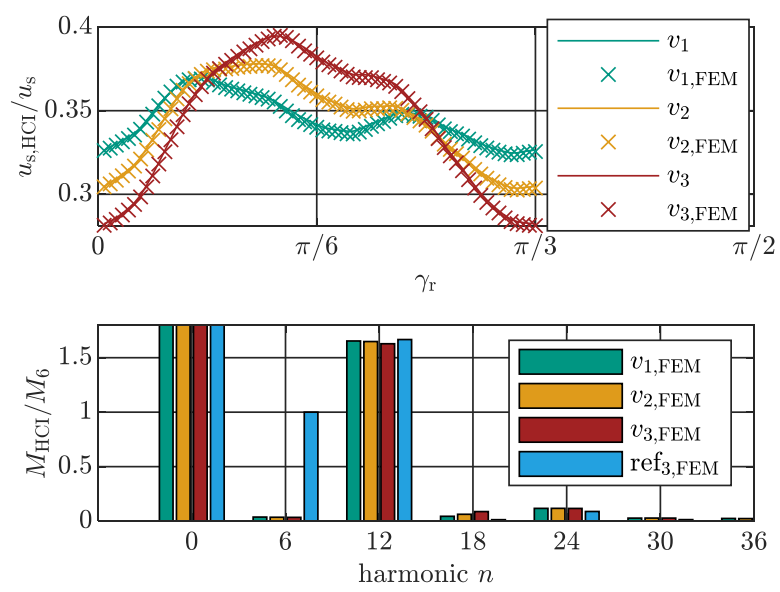

Fig. 9: Comparison in induced voltages (top) between model and FEM for the three current trajectories found in Fig. 8 and the resulting FEM torque (bottom) for the same currents 


\section{A. HCI in dq-plane}

To find the optimal HCI trajectories the proposed method is now applied to the entire dq-plane with two different optimization objectives:

- $\quad$ A: find $\alpha(\mathrm{d}, \mathrm{q}), \gamma(\mathrm{d}, \mathrm{q})$ for which $\min \left\{u_{\mathrm{s}}(t)\right\}$

- $\quad$ B: find $\alpha(\mathrm{d}, \mathrm{q}), \gamma(\mathrm{d}, \mathrm{q})$ for which $\min \left\{i_{n=6}(t)\right\}$

Objective A searches for the $\alpha$ and $\gamma$ for each operating point in the dq-plane which minimizes the maximum induced voltage $u_{\mathrm{s}}$ within an electrical period. Objective B searches for the $\alpha$ and $\gamma$ for each operating point in the dq-plane which minimizes the harmonic current amplitude $i_{n=6}$ within an electrical period.

The results gained in this way are saved. Four matrices can be saved for each optimization objectives. These four result matrices consist of $\boldsymbol{\alpha}(\mathrm{d}, \mathrm{q}), \boldsymbol{\gamma}(\mathrm{d}, \mathrm{q}), \boldsymbol{i}_{\boldsymbol{n}}(\mathrm{d}, \mathrm{q}), \boldsymbol{\varphi}_{\mathrm{c}}(\mathrm{d}, \mathrm{q})$. Since different angles are stored here, it may be more practical to convert these four matrices for later transfer to a controller or postprocessor. Another possibility to express a trigonometric function is to express it as sine and cosine function similar to (2). The results stored in this way are then continuously in the dq-plane.

For $R=0$ the result matrices are valid for all speeds, because in (12) and (13) the time derivative can be modified to:

$$
\frac{\mathrm{d}}{\mathrm{d} t}=\frac{\mathrm{d}}{\mathrm{d} t} \frac{\mathrm{d} \gamma}{\mathrm{d} t} \frac{\mathrm{d} t}{\mathrm{~d} \gamma}=\omega \cdot \frac{\mathrm{d}}{\mathrm{d} \gamma}
$$

This means that both terms contain $\omega$ and can be excluded. The change in flux linkage per angle is the same at any speed, if eddy currents and iron losses are neglected. Therefore, the speed of rotation has no influence on the location of the found minimum expressed in $\alpha$ and $\gamma$.

\section{B. $\mathrm{HCI}$ in $\mathrm{M} / \mathrm{n}$-plane}

There are now two different rules A and B how to execute $\mathrm{HCI}$ in the dq-plane. In order to calculate the maximum torque over the whole speed range a further optimization
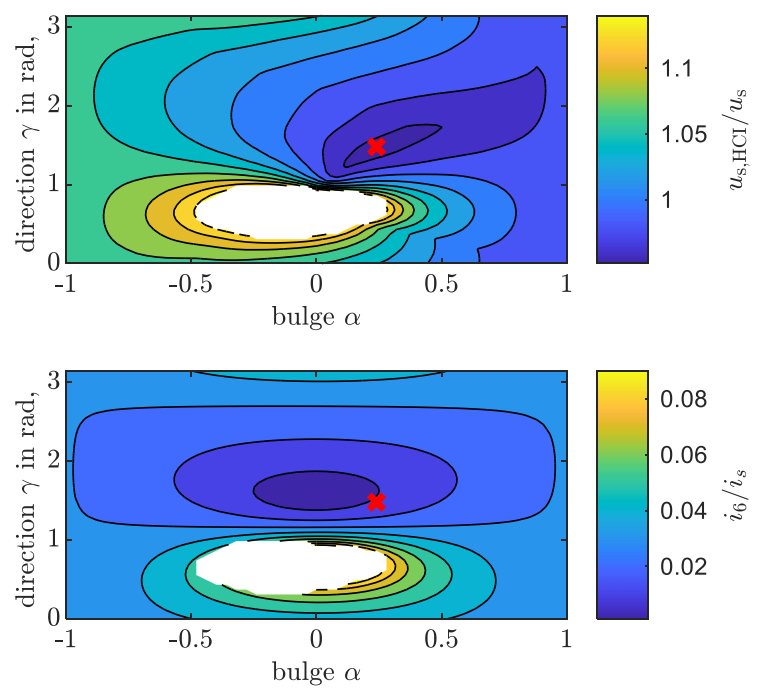

Fig. 10: Resulting maximum voltage (top) and used harmonic curren amplitude (bottom) for all possible current trajectories in $\gamma \alpha$-plane which eliminate the $6^{\text {th }}$ torque harmonic at the operation point shown in Fig. 2, the combination with the lowest induced voltage is marked (red) calculation is necessary. This time the objective function is to maximize the torque under certain constraints and HCI rules. The usual constraints are that a certain maximum current $i_{\mathrm{s}, \max }$ and a certain maximum voltage $u_{\mathrm{s}, \max }$ must not be exceeded. The reference curve $M(n)$ does not use HCI and is called 0 . Two additional $M(n)$ curves are calculated using HCI strategy A and HCI strategy B.

The currents for which the torque becomes maximum and fulfill the mentioned conditions for increasing speed are shown in Fig. 11. This shows that the curves with HCI must reduce the maximum current earlier and further due to the voltage limit. Moreover, it can be seen that method A performs better than method B on the limit curve. This effect is also clearly visible in Figure 12.

The maximum torque over speed for HCI strategy A and $\mathrm{B}$ is illustrated in Figure 12. As a reminder, strategy $\mathrm{A}$ is optimized for the lowest possible induced voltage and strategy $\mathrm{B}$ is optimized for the lowest possible current harmonic. To emphasize the difference, the resulting torque profile is normalized with the maximum torque curve without HCI. Also the speed is normalized with the speed at the corner to the field weakening area.

Overall, the achievable torque with $\mathrm{HCI}$ is lower, with a few exceptions. The HCI strategies hardly differ up to one speed. From the speed at which we reach the voltage limit, HCI strategy A performs significantly better. The torque advantage is up to $8 \%$.

In the speed range from 0 to 1 the maximum current amplitude limits the torque. Compared to a control strategy not utilizing HCI, HCI requires a higher current amplitude, therefore the maximum torque is lower. This means that $2 \%$ of the possible torque is invested in a smoother torque.

Above the speed of 1 the voltage is the limiting factor. Here strategy A benefits from the fact that the current trajectories were selected which also eliminate the $6^{\text {th }}$ torque harmonic, but also minimize the induced voltage. Up to a speed of 2, strategy A even comes close to the original maximum torque, or exceeds it in part. This is because when calculating the maximum voltage, the harmonics in the induced voltage are taken into account. Both the harmonics from the flux linkage and from the injected current. So if strategy A exceeds the original torque, the injected current attenuates a harmonic in the induced voltage and the normalized torque becomes $>1$.

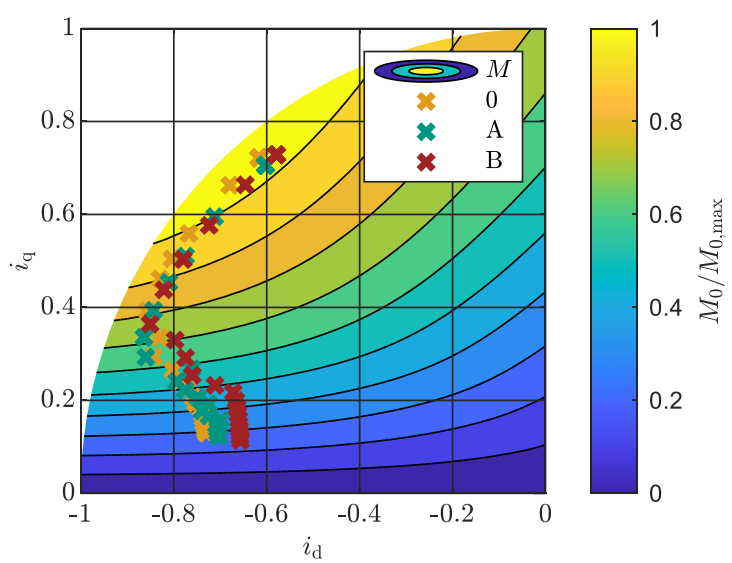

Fig. 11: Torque map over $i_{\mathrm{d}}$ and $i_{\mathrm{q}}$ with maximum values for increasing speed for no $\mathrm{HCI} 0$ and the $\mathrm{HCI}$ strategies A and B 


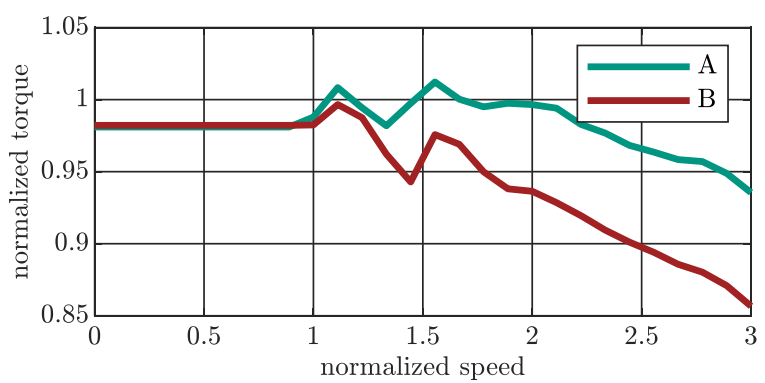

Fig. 12: Maximum torque profile over speed for HCI strategy A and B, here the torque is normalized to the torque curve without HCI, the speed is also normalized to the characteristic speed

At speeds above 2 this effect is masked by the high induced voltage of the current harmonic and both strategy $\mathrm{A}$ and B lose torque. Nevertheless, strategy A performs up to $8 \%$ better.

As an overall strategy, one can proceed as follows. To minimize ohmic losses, use HCI strategy B for the largest range in the torque speed map. Above the maximum characteristic curve of $\mathrm{B}, \mathrm{HCI}$ strategy $\mathrm{A}$ is used. Above this maximum characteristic curve, no HCI can be used with regard to current and voltage limits.

\section{CONCLUSION}

In this paper a model for the torque pulsation in PMSM was presented. It was shown how an injected harmonic in the d- and q- current modifies the torque. A novel $\gamma \alpha$-plane was introduced to model any current trajectory. A method is proposed to find a current which minimizes a harmonic in the torque for each possibility in the $\gamma \alpha$-plane. From all solutions two are selected. The first strategy is to choose the trajectory that minimizes the current demand $i_{n}$, the second one is minimizing the induced voltage $u_{\mathrm{s}}$. This method was applied to all operating points and all speeds. In a comparison of the two strategies it could be shown that the applicability of HCI on the maximum torque / speed characteristic benefits from the second strategy. The range in the torque / speed map to which HCI can be applied is thus increased. All model-based findings were validated by means of FEA calculations.

Considerations not yet addressed are the influence of the current trajectories on the iron losses. These could lead to further optimization objectives. Although it was mentioned how the identified solution matrices can be stored, an implementation in a controller still needs to be investigated. In particular, the required high frequencies, in measurement data acquisition, processing and conversion must be considered more thoroughly. This and a validation by measurements will be part of future research.

\section{REFERENCES}

[1] T. H. Pham, P. Wendling, P. Lombard, T. M. Jahns, and W. L. Soong, "Pulsating torque minimization techniques for permanent magnet AC motor drives - A review," IEEE Trans. Ind. Electron., vol. 43, no. 2, pp. 3-8, 1996.

[2] L. Zhu, S. Z. Jiang, Z. Q. Zhu, and C. C. Chan, "Analytical methods for minimizing cogging torque in permanent-magnet machines," IEEE Trans. Magn., vol. 45, no. 4, pp. 2023-2031, 2009.

[3] P. H. Mellor, R. Wrobel, N. McNeill, and D. Drury, "Impact of winding and rotor design on efficiency and torque ripple in brushless AC permanent magnet traction motors," IET Conf. Publ., no. 538 CP, pp. 240-244, 2008.

[4] Z. Azar, Z. Q. Zhu, and G. Ombach, "Influence of electric loading and magnetic saturation on cogging torque, Back-EMF and torque ripple of PM machines," IEEE Trans. Magn., vol. 48, no. 10, pp. 2650-2658, 2012

[5] M. Halwas et al., "Coherences between production technology and performance of electric traction drives," 2019 9th Int. Electr. Drives Prod. Conf. EDPC 2019 - Proc., 2019.

[6] T. H. Pham, P. Wendling, and P. Lombard, "Current Harmonics Injection Table To Minimize Torque Ripples For Electric Powertrain Interior Permanent Magnet Motor Over Maximum Torque- Speed Curve," Proc. Int. Conf. Power Electron. Drive Syst., vol. 2019-July, pp. 3-8, 2019.

[7] M. Harries, M. Hensgens, and R. W. De Doncker, "Noise Reduction Via Harmonic Current Injection for ConcentratedWinding Permanent Magnet Synchronous Machines," ICEMS 2018 - 2018 21st Int. Conf. Electr. Mach. Syst., pp. 1157-1162, 2018.

[8] A. Najmabadi, W. Xu, and M. Degner, "A sensitivity analysis on the fifth and the seventh harmonic current injection for sixth order torque ripple reduction," 2017 IEEE Int. Electr. Mach. Drives Conf. IEMDC 2017, 2017.

[9] J. Richter, Modellbildung, Parameteridentifikation und Regelung hoch ausgenutzter Synchronmaschinen. 2016.

[10] G. Štumberger, B. Štumberger, and T. Marčič, "Magnetically nonlinear dynamic models of synchronous machines and experimental methods for determining their parameters," Energies, vol. 12, no. 18, pp. 1-22, 2019.

[11] S. Decker et al., "DQ0 Modelling and Parameterization of small Delta connected PM Synchronous Machines," 2020, in press.

[12] G. Heins, M. Thiele, and T. Brown, "Accurate torque ripple measurement for PMSM," IEEE Trans. Instrum. Meas., vol. 60, no. 12, pp. 3868-3874, 2011. 